\title{
Inherited epilepsy linked to gangliosides
}

A rare form of familial epilepsy has been linked to a mutation in a gene that is normally involved in the production of gangliosides in the brain, according to new research published in Nature Genetics. Gangliosides are glycolipids that contain sialic acid and are found in almost all cells, but are especially abundant in the central nervous system. Abnormalities in their degredation are usually associated with lysosomal storage disorders, but, so far, their role in the brain has not been well characterized. This new work indicates that they might be important for regulating neuronal hyperexcitability.

Inherited epilepsies are thought to account for $\sim 40 \%$ of cases. A small proportion of these are related to single gene defects, several of which have been shown to encode ion channels. Simpson et al. have identified a different mechanism underlying inherited epilepsy in two branches of a large family with an autosomal recessive form of epilepsy who were connected through a fifth-generation common ancestor. Eight surviving individuals were affected with severe epilepsy that began in the first year of life, and mainly involved generalized tonic clonic (grand mal) seizures that were not associated with any focal abnormalities on electroencephalography (EEG) and MRI. The onset of epilepsy was accompanied by a marked decline in motor and verbal development, as well as severe visual impairments.

Genome-wide linkage analysis showed that all affected children had a single region of homozygosity on

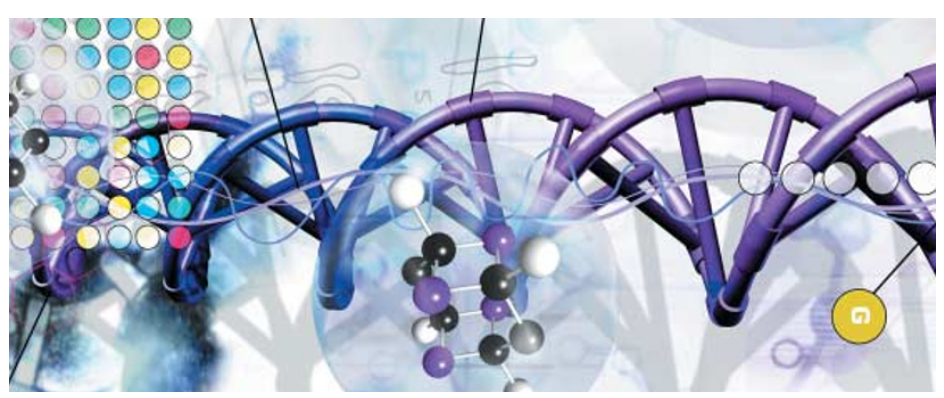

chromosome 2 that was not found in unaffected siblings or parents. A nonsense mutation (C694T) in the gene SIAT9I was identified with highthroughput sequencing, and it is thought that this mutation abolishes the activity of GM3 synthase. GM3 synthase triggers the first step in the production of central nervous system gangliosides by transferring a sialic acid residue to lactosylceramide to form GM3, the main ganglioside in human plasma. Disrupting the activity of GM3 synthase therefore prevents the formation of GM3 and other important gangliosides.

Biochemical analyses showed that GM3 and its derivatives were completely absent in the affected members of the family, although they did have elevated levels of its immediate precurser, lactosylceramide. It is not clear, however, whether the epilepsy results from reduced GM3 levels or from elevated lactosylceramide levels. Nevertheless, this research highlights a novel mechanism that might, in the future, help us to understand other forms of epilepsy. Moreover, the involvement of the ganglioside-formation pathway in epilepsy indicates that these glycolipids might be important for regulating neuronal excitability.

Alison Rowan

\section{(2) References and links} original ReSEARCh PAPER Simpson, M. A. et al. Infantile-onset epilepsy syndrome caused by a homozygous loss-of-function mutation of GM3 synthase. Nature Genet. 36, 1225-1229 (2004) FURTHER READING Steinlein, O. K. Genetic mechanisms that underlie epilepsy. Nature Rev. Neurosci. 5, 400-408 (2004)

\section{IN BRIEF}

CELL BIOLOGY OF THE NEURON

Truncated TrkB receptor-induced outgrowth of dendritic filopodia involves the p75 neurotrophin receptor.

Hartmann, M. et al. J. Cell Sci. 117, 5803-5814 (2004)

Truncated splice variants of the TrkB neurotrophin receptor are abundant in the adult rodent CNS, but little is known about their physiological functions. Hartmann et al. present evidence that the TrkB.T1 isoform interacts with the $\mathrm{p} 75^{\mathrm{NTR}}$ receptor, thereby initiating a signalling cascade that induces outgrowth of dendritic filopodia. This could be one of the mechanisms that contribute to the formation of new synapses in the postnatal CNS.

\section{CIRCADIAN RHYTHMS}

Circadian gene expression in individual fibroblasts: cellautonomous and self-sustained oscillators pass time to daughter cells.

Nagoshi, E. et al. Cell 119, 693-705 (2004)

The body's circadian rhythms are centrally controlled by the suprachiasmatic nucleus (SCN) in the brain, and oscillators in peripheral tissues were originally thought to lose their rhythms rapidly in the absence of SCN input. However, Nagoshi et al. now show that oscillations in circadian gene expression are sustained in a cell-autonomous manner in cultured fibroblasts, and although cell division causes a phase shift in the circadian cycle, the oscillations also persist in the daughter cells.

\section{SENSORY SYSTEMS}

\section{Magnetoreception and its trigeminal mediation in the} homing pigeon.

Mora, C. V. et al. Nature 432, 508-511 (2004)

This study provides new evidence to support the idea that homing pigeons use magnetoreception to find their way home. Mora et al. were able to train pigeons to detect a magnetic field anomaly, but this ability was impaired by anaesthetizing the upper beak or attaching a magnet to it, or by severing the ophthalmic branch of the trigeminal nerve. The authors suggest that magnetite in the upper beak region is used to detect the magnetic field and the trigeminal nerve relays this information to the brain.

\section{STEM CELLS}

Chromatin remodeling and histone modification in the conversion of oligodendrocyte precursors to neural stem cells.

\section{Kondo, T. \& Raff, M. Genes Dev. 18, 2963-2972 (2004)}

Purified oligodendrocyte precursor cells (OPCs) can be converted to multipotent neural stem-like cells (NSLCs) that generate both neurons and glia, so they could provide a source of neurons for CNS repair. To investigate how this potential might be harnessed, Kondo and Raff analysed the molecular mechanisms that underlie the conversion of OPCs to NSLCs. They show that this process depends on the reactivation of Sox 2 gene expression, which in turn depends on chromatin remodelling at the Sox 2 promoter. 